Paul Ulrich Unschuld

\title{
Gesundheitspolitische Herausforderungen im Kontext der Koexistenz von Schulmedizin und alternativen Heilweisen
}

$\mathrm{Ob}$ man dies nun für gut erachtet oder nicht, Tatsache ist, dass die weitgehende Monopolstellung der um eine naturwissenschaftliche Begründung bemühten medizinischen Versorgung der Bevölkerung in den Gesundheitssystemen aller westlichen Industrienationen zunehmend von einer transkulturellen Vielfalt der heilkundlichen Angebote infrage gestellt wird. Unterschiedliche Theorien des Krank- und Gesundseins und der angemessenen Therapie sowie daraus abgeleitete therapeutische Massnahmen aus der eigenen europäischen Kultur ebenso wie Importe aus nichtwestlichen Kulturen bilden gemeinsam mit der sogenannten Schulmedizin einen grossen Markt, auf dem die "Verbraucher» sich aus verschiedensten Gründen im Bedarfsfall das auswählen, was sie als sinnvoll und hilfreich ansehen.

Diese Vielfalt des Gesundheitswesens ist eine unvermeidbare Begleiterscheinung der Umwandlung moderner Gesellschaften von einer sehr begrenzten Anzahl weltanschaulich weitgehend monolithischer Blöcke zu einer fast unüberschaubaren Diversifizierung der Daseinsdeutung und Lebensentwürfe. Auf verschiedenen Ebenen, nicht zuletzt auch in den Tagesmedien, wird eine zum Teil sehr engagierte Diskussion geführt zwischen denen, welche die Monopolstellung der Schulmedizin auch für die Zukunft gesichert sehen möchten, und denen, die auch ausserhalb der an die Naturwissenschaften angelehnten Medizin ein ernstzunehmendes heilkundliches Potenzial vermuten oder entdeckt haben. Solche Diskussionen sind Ausweis einer Übergangsperiode, in der noch kein gesellschaftlicher Konsens gefunden ist, wie mit der neuen Heterogenität umzugehen ist. Diesen Konsens zu finden, ist eine der nicht unwichtigen Aufgaben, die den Übergang in die gesellschaftliche Heterogenität begleiten werden.

Von einer neutralen, übergeordneten Warte aus gesehen gilt es angesichts der gegenwärtigen, nicht selten auch polemisch bestimmten Kontroversen, gesundheitspolitisch und verantwortungsvoll zunächst einmal die Werte zu bestimmen, die in unserer Gesellschaft im Umgang mit Kranksein und Gesundheit massgebend sein sollen, und sodann daraus geeignete Strukturen abzuleiten, welche die Umsetzung dieser Werte in der täglichen Praxis zu erstreben oder gar zu realisieren geeignet sind.

Grundlegend ist für das Gesundheitswesen in Deutschland nach wie vor der Solidaritätsgedanke. Solidarität bedeutet, dass in einer Gemeinschaft, die sich realen oder möglichen Gefahren gegenübersieht, alle füreinander einstehen. Solidarität ist nicht mit den christlichen Werten von Barmherzigkeit oder Mitleid zu verwechseln. Solidarität entsteht aus der nüchternen Erkenntnis, dass in einer Gemeinschaft jeder Mensch von einer
Gefahr in der Weise heimgesucht werden kann, dass er als Einzelner nicht allein imstande ist, diese Heimsuchung zu meistern. Der Einzelne bedarf der Unterstützung der anderen. Aus diesem Grund wird in einem solidarischen Versicherungssystem von jedem Gemeinschaftsmitglied ein Pflichtbeitrag erhoben (diejenigen, die nicht fähig sind, einen solchen zu leisten, können aus Barmherzigkeit von Beiträgen freigestellt werden). Die so gesammelten Mittel stehen im Bedarfsfall jedem Bedürftigen zur Verfügung. Ein solches Solidarsystem kann sich nur solange halten, wie eine wichtige Bedingung erfüllt bleibt: Das von jedem Gemeinschaftsmitglied pflichtgemäss entrichtete Geld muss auf der Grundlage einer von allen geteilten Vernunft verwaltet und im Bedarfsfall eingesetzt werden. Mit anderen Worten: Die als Pflichtbeiträge von jedem Gemeinschaftsmitglied entrichteten und nun der Solidarkasse zur Verfügung stehenden finanziellen Mittel dürfen nur für Massnahmen (im Gesundheitswesen sind dies diagnostische und therapeutische Massnahmen) ausgegeben werden, deren Sinn und Wert von allen Gemeinschaftsmitgliedern eingesehen werden. Daraus entsteht ganz deutlich eine Trennung zwischen den heilkundlichen Therapien, die vom Solidarsystem finanziert werden dürfen, und jenen, die nicht finanziert werden können. Selbstverständlich

\section{KARGER}

(C) 2016 S. Karger GmbH, Freiburg

Fax +497614520714
Prof. Dr. phil. Dr. phil. habil. Dr. med. habil. M.P.H. Paul Ulrich Unschuld Horst-Görtz-Stiftungsinstitut für Theorie, Geschichte und Ethik Chinesischer Lebenswissenschaften

Charité - Universitätsmedizin Berlin, Campus Mitte

Charitéplatz 1, 10117 Berlin, Deutschland

unschuld@charite.de 
bleibt es allen gesetzlichen Krankenkassen freigestellt, attraktive, spezifische Tarife auch für solche Versicherte zu entwerfen, die sich für wissenschaftlich nicht legitimierbare Therapien entscheiden.

Das einzige Paradigma, das in einer modernen Industriegesellschaft von allen Menschen in Anspruch genommen und somit kollektiv akzeptiert wird, ist das Paradigma der modernen Naturwissenschaften und der aus diesen abgeleiteten Technologien. Wir sprechen hier von einer vollkommenen Kollektivsicht. Kein Mensch kann sich diesem Paradigma entziehen. Kein Mensch vermag ein Leben ausserhalb des naturwissenschaftlichtechnologischen Paradigmas zu führen. Eine vergleichbare vollkommene Kollektivsicht trifft auf kein einziges anderes weltanschauliches Paradigma $\mathrm{zu}$, sei es nun eine Religion oder seien es die säkularen relationistischen Erklärungsmodelle der chinesischen Yin-Yang- und Fünf-Phasen-Lehren, ganz zu schweigen von Partialparadigmen, wie z.B. der homöopathischen Leitlehre «similia similibus curentur». Jedem Mitglied einer modernen Gesellschaft steht es frei, sich einer dieser Lehren anzuschliessen oder sich davon fernzuhalten. Ein Leben kann so oder so geführt werden. Es handelt sich hier um Binnensichten von Teilgruppen einer Gesellschaft im Gegensatz zu der Kollektivsicht, die von der Gesellschaft insgesamt geteilt wird. Heilkundliche Massnahmen, die aus einer Binnensicht erwachsen, sind somit für eine Teilgruppe der Gemeinschaft sinnvoll und handlungsbestimmend; für andere sind sie es wiederum nicht.

Folglich gilt in einer Solidargemeinschaft das Prinzip: Binnensicht verlangt Binnenfinanzierung. Heilkundliche Massnahmen, die aus einer Binnensicht erwachsen, sind mit den Geldern, die durch Pflichtbeiträge aller Mitglieder der Solidargemeinschaft angesammelt wurden, nicht zu finanzieren und müssen von den Anhängern der Binnensicht selbst getra- gen werden. Sie können, so sie dies möchten, eine eigene Solidargemeinschaft auf der Grundlage der nur von ihnen geteilten Weltanschauung bilden - in derselben Weise wie in einer Gesellschaft kleine religiöse Gemeinschaften eigene Solidargemeinschaften bilden. In dem Moment, in dem aus Pflichtbeiträgen solche Therapien finanziert werden, die einem grösseren Teil der Solidargemeinschaft unvernünftig erscheinen, also für sinnlos erachtet werden, ist der Zusammenhalt der Solidargemeinschaft gefährdet; die Gemeinschaft wird über kurz oder lang zerbrechen. Menschen, die keine gemeinsame Wertegrundlage haben, bilden keine Solidargemeinschaften. Eine solche Entwicklung ist in der Gegenwart zu beobachten.

Das Prinzip der Solidarität im Gesundheitswesen ist durch die zunehmende weltanschauliche und kulturelle Heterogenität auch der Gesellschaft gefährdet. Es gilt, zumindest ein Bemühen zu zeigen, es auch in einer Zeit aufrechtzuerhalten, in der nicht nur die zunehmende kulturelle Heterogenität die Solidargemeinschaft gefährdet, sondern auch die kommerziellen und ökonomischen Massstäbe einer Gesundheitswirtschaft, die immer stärker zur bestimmenden Kraft von Vorbeugung und Therapie des Krankseins werden [1].

$\mathrm{Zu}$ den Grundwerten der Solidarität gesellt sich nun ein zweiter Grundwert, und zwar jener der Therapiefreiheit. Kranksein, Behinderung und die Gefahr eines verfrühten Todes sind für fast alle Menschen emotional höchst belastende Erfahrungen. Ihnen zur Seite stehen Verwandte, Freunde, vielleicht Kollegen, die diese emotionalen Belastungen mittragen und nach Auswegen aus der Krise suchen. Ihnen gegenüber stehen in der Schulmedizin Fachleute, die ihre Diagnosen und Therapien nüchtern und emotionslos auf der Grundlage ihrer Ausbildung ausgehend von den Befunden durchzuführen angehalten sind. Es mag sein, dass Emotion und Nüchternheit, wenn sie in einer solchen
Situation aufeinander treffen, leicht zueinander finden und gemeinsam den rechten Weg einschlagen, der beide Seiten zufriedenstellt - den Kranken und dessen Umfeld einerseits und den Therapeuten andererseits. Das muss jedoch nicht sein: Es ist nicht selten der Fall, dass das Handeln der Leidenden und ihres Umfelds nicht durch nüchterne Statistiken, sondern von ihren emotionalen Ängsten und ihrer Zuversicht geleitet wird. Hier stellt sich die nächste Frage dahingehend, in welchem Masse und innerhalb welcher Grenzen den Patienten und deren Umfeld in einer modernen, weltanschaulich und somit kulturell heterogenen Gesellschaft eine Therapiefreiheit gewährt werden kann.

Exakte Studienergebnisse sind dazu nicht vorhanden, aber der Blick in die Geschichte und in die Gegenwart zeigt, dass es für einen Grossteil der Beschwerden, derentwegen Menschen medizinischen oder heilkundlichen Beistand suchen, unerheblich ist, mit welchen theoretischen Ansätzen und praktischen Massnahmen sie behandelt werden. Sie fühlen sich besser oder sogar wieder gesundet, und dieser Erfolg kann mit der Homöopathie, mit Bachblüten, mit indischem Ayurveda oder einer der unzähligen Spielarten angeblich Chinesischer Medizin erreicht werden. Ob man dies nun als Placebo bezeichnet oder nicht, Tatsache ist, dass Menschen eine Linderung oder gar Heilung vieler ihrer Leiden empfinden, nachdem sie sich dorthin gewandt haben, wo sie Hilfe zu finden erhoffen und wo sie erwarten, dass sie dort auch eine Heilung erfahren können - aus welchen Gründen auch immer.

Daraus leitet sich der Schluss ab, dass es eine Beschneidung persönlicher Freiheit sein kann und gerade bei einigen der vielen Leiden, die sich dem naturwissenschaftlichen Deutungsmuster entziehen, vielleicht sogar kontraproduktiv ist, wenn man Menschen gegen ihre Empfindungen in eine heilkundliche Therapie drängt 
oder zwingt, die ihnen bedrohlich oder auch einfach bedeutungslos zu sein scheint.

Aus dieser Anerkennung des hohen Wertes der Therapiefreiheit kann freilich ein Konflikt erwachsen mit den Forderungen, die aus dem ebenso hohen Wert der Solidarität und der daraus folgenden Beschränkung der durch eine Solidarkasse zu finanzierenden Therapien hervorgehen.

Die Frage ist, wie man diese beiden Werte in einem Gesundheitswesen miteinander vereinen kann. Eine denkbare Lösung bestünde darin, verschiedene Tarifstrukturen einzuführen. Diejenigen Mitglieder der Solidargemeinschaft, die Pflichtbeiträge entrichten, müssen davon ausgehen können, dass die daraus entstandenen finanziellen Mittel allein für Therapien auf der Grundlage der kollektiv akzeptierten Paradigmen - das sind die Naturwissenschaften und die daraus abgeleiteten Technologien - eingesetzt werden. Für diejenigen, die darüber hinaus Therapien in Anspruch nehmen möchten, deren Sinnhaftigkeit allein der Binnensicht einer begrenzten Bevölkerungsgruppe verhaftet ist, sind Beitragsstrukturen zu entwickeln, die diesem Anliegen Rechnung tragen. Es ist nicht ausgeschlossen, dass sich bei genauem Hinschauen zeigt, dass solche Patienten sogar geringere Krankheitskosten verursachen als die allein der Schulmedizin Vertrauenden. Belastbare Forschungsergebnisse zur Beantwortung dieser Frage sind nicht verfügbar. Auf diese Weise sind Solidarität und Therapiefreiheit vereinbar. Ob die finanziellen Belastungen letztlich unterschiedlich oder ausgeglichen sind, muss sich in entsprechenden Beobachtungen herausstellen.

Das leitet über zu einem dritten Grundwert, den es aufrechtzuerhalten gilt, und das ist die Sicherheit. Vielleicht die wichtigste Aufgabe gemeinschaftlicherOrgane, also der Regierung und ihrer Behörden, liegt in der Gewährung von Sicherheit für alle Bürger. Das ist die Sicherheit vor äusseren
Bedrohungen ebenso wie die Sicherheit vor Bedrohungen aus dem Inneren der Gesellschaft. Im Gesundheitswesen bedeutet das den Schutz jedes Einzelnen vor Massnahmen, die zu einer körperlichen oder seelischen Schädigung führen, und somit auch vor Anwendern, die den menschlichen Organismus nicht ausreichend kennen und sich fälschlicherweise die Anwendung diagnostischer und therapeutischer Eingriffe zutrauen, die sich anders als erwartet als der Gesundheit der ihnen anvertrauten Patienten abträglich erweisen. Das gesamte Medizinstudium und auch zahlreiche Strukturen zur Überwachung medizinischer Praxis sind dem Streben nach Sicherheit geschuldet.

Hier lautet nun eine weitere Frage: Wie steht es um die Sicherheit, und insbesondere die Qualitätssicherung, bei der Anwendung von Heilverfahren, die sich der naturwissenschaftlichen, insbesondere aber der quantitativen und qualitativen Bewertung durch Dritte weitgehend entziehen? Die Gefahr liegt hier zum einen in der Fehldiagnose einer schwerwiegenden körperlichen oder seelischen Störung als nicht so schwerwiegend, dass sie nicht doch mit den Methoden nichtnaturwissenschaftlich legitimierter Heilverfahren therapiert werden könnte, die dieser Störung aber tatsächlich nicht gewachsen sind. Die Gefahr liegt zum zweiten in der Fortsetzung einer untauglichen «alternativen» Behandlung bis in eine Situation, in der das Leiden für den Patienten eine irreversibel bedrohliche Phase erreicht hat. Die Gefahr liegt drittens in der Anwendung von therapeutischen Massnahmen, die eine direkte Gefährdung der Gesundheit des zu Behandelnden bewirken. Ist es also Aufgabe des Staates, hier Grenzen zu ziehen und Aufsicht auszuüben, und wenn dem so ist, wo müssen diese Grenzen gezogen werden und wie weit muss die Aufsicht reichen?

Tatsache ist: Die Schulmedizin hat im Laufe ihrer Entwicklung bis in die jüngste Zeit unzählige zuvor nie er- hoffte Erfolge erzielt, aber auch viele Schäden verursacht. Die Anwender alternativer Heilverfahren können auf viele zufriedene Patienten verweisen, aber einige ihrer therapeutischen Massnahmen sind mindestens risikoreich. Tatsache ist, dass kein Heilsystem ausserhalb der Schulmedizin die Möglichkeit bietet, messbare, also durch Dritte nachprüfbare Daten über den Gesundheitszustand bzw. über die Krankheit eines Patienten zu erheben und damit eine Aufsicht über angemessenes oder unangemessenes, sachgerechtes oder unsachgerechtes therapeutisches Vorgehen auszuüben. Im Rahmen der alternativen Heilweisen kann eine solche Aufsicht bestenfalls durch Gleichgesinnte, d.h. Teilhabende an einer Binnensicht, durchgeführt werden. Die Objektivität, die allein quantifizierende Messungen ermöglicht, ist niemals gegeben.

Es fehlen somit auch bei allen schon genannten alternativen Heilverfahren Kriterien, nach denen die Kompetenz eines angeblichen Heilkundigen überprüft werden könnte. Man kann einem solchen Heilkundigen in einem längeren oder kürzeren Lehrgang viele der in dem entsprechenden Heilsystem enthaltenen Deutungen bestimmter Leidenszustände vermitteln und diese dann als Ausweis der Kompetenz auch wieder abfragen. Im Unterschied zu der Schulmedizin ist jedoch keinerlei Gewähr gegeben, dass diese Deutungen, die sich auf Diagnose und Therapie auswirken, tatsächlich irgendeinen nachprüfbaren Bezug zu physiologischen oder pathologischen Vorgängen im menschlichen Organismus haben. Eine Qualitätssicherung ist daher allein unter Gleichgesinnten denkbar und daher für Aussenstehende irrelevant.

Das Prinzip der Sicherheit kann folglich nur sehr begrenzt auf derlei Heilkundige angewendet werden. Es bieten sich etwa eine Pflichtausbildung z.B. in menschlicher Morphologie und in der Erkennung von übertragbaren Krankheiten sowie ein Verbot invasiver diagnostischer und 
therapeutischer Eingriffe, die bei inkompetenter Anwendung Schaden verursachen können, an. Doch hier entstünde ein Problem. Ist es angemessen in einer modernen Gesellschaft, in der viel von Patientensouveränität und von mündigen Patienten die Rede ist, solche Anforderungen an all diejenigen zu stellen, die sich, ohne ein Medizinstudium durchlaufen zu haben, eine Heilkunde, die ausserhalb des naturwissenschaftlichen Paradigmas steht, aneignen und im Rahmen der Therapiefreiheit anwenden möchten? Kann man nicht vielleicht voraussetzen, dass ein Hilfesuchender die staatlich beaufsichtigte Schulmedizin als das ansieht, was im Strassenverkehr der mit einer Ampel und durch Zebrastreifen gekennzeichnete, in der Regel sichere Überweg über eine gefährliche Hauptverkehrsstrasse ist? Wer ausserhalb dieses Überwegs quer über die Strasse läuft, der geht bewusst ein persönliches Risiko ein, das möglicherweise zu Verletzungen oder gar in den Tod führt. Vertraut ein mündiger Patient sich einem solchen Risiko an, dann liegt ein unguter Ausgang allein in seiner Verantwortung und in jener des konsultierten heilkundlichen Praktikers, der gleichsam als Lotse den eine Abkürzung Suchenden auf die ungesicherte Strasse «gelockt» hat und somit mitschuldig ist an diesem Ausgang. Bleiben wir bei dem recht geeigneten Vergleich mit dem gesicherten und dem ungesicherten Strassenübergang. Dass einige Menschen den gesicherten Zebrastreifen missachten und an ungesicherter Stelle über die Hauptverkehrsstrasse laufen, rechtfertigt es noch nicht, dass die Gesellschaft nun entlang aller Strassen hohe Zäune errichtet, damit keiner auf den Gedanken kommt, sich einer Gefahr auszusetzen, nur weil er/sie es eilig hat. Ähnlich erscheint es wohl sinnvoll, mündige Gesellschaftsmitglieder auf die unterschiedlichen Aufsichtsstrukturen und Sicherungsmechanismen aufmerksam zu machen, die für die Schulmedizin auf der einen und die «alternativen» Heilverfahren auf der anderen Seite möglich sind, und es dann diesen mündigen Bürgern selbst zu überlassen, wohin ihr Vertrauen sie führt.

Der Staat nimmt in manchen Bereichen seine Verantwortung für die Sicherheit der Bürger ernst, in anderen Bereichen jedoch auch nicht. Ein Beispiel ist die Freiheit, die der Lebensmittelindustrie eingeräumt ist, viele nachweislich gesundheitsschädliche «Lebensmittel» mit aggressiver Werbung auf dem Markt einzuführen. Für die negativen gesundheitlichen Folgen muss dann in der Regel die Solidarkasse der Pflichtversicherten aufkommen - ein höchst problematischer Zustand. Solange solche eklatanten Lücken in den Anstrengungen der staatlichen Organe, der Bevölkerung Sicherheit im Inneren wie im Äusseren zu gewähren, nicht gefüllt werden, solange ist der sehr viel weniger problematische Bereich der Therapiefreiheit nur von zweitrangiger Bedeutung. Der Werbung für gesundheitsgefähr- dende «Lebensmittel» kann sich kaum jemand entziehen; die möglichen Konsequenzen einer Entscheidung des mündigen Patienten für oder wider die Schulmedizin oder ein alternatives Vorgehen sind vergleichsweise einfach aufzuzeigen.

Die gesundheitspolitische Herausforderung besteht darin, ein Gesundheitswesen zu schaffen, das die genannten drei Werte in die Praxis umzusetzen geeignet ist. Dies ist ein Gesundheitswesen, das der Entwicklung moderner Gesellschaften hin zu weltanschaulicher Vielfalt, die sich auch auf die Deutung von Kranksein und Gesundheit auswirkt, Rechnung trägt. Es handelt sich um ein Gesundheitswesen, das der Tatsache Rechnung trägt, dass weder die Schulmedizin noch ein alternatives Heilverfahren unfehlbar ist oder auf jeden Zustand des Krankseins eine passende Therapie einleiten könnte. Folglich ist es allein sinnvoll, alle Ressourcen zu nutzen - wenn das Ziel des Gesundheitswesens darin besteht, den Hilfesuchenden ohne ideologische Scheuklappen Hilfe zu gewähren. Das mag selbstverständlich klingen, ist es aber nicht in einer Zeit, in der kommerzielle Erwägungen zunehmend den diagnostischen Aufwand und die medizinische Therapieentscheidung beeinflussen und in der sich Renditeund Amortisationsziele privatwirtschaftlicher ebenso wie kommunaler Anbieter von Gesundheitsdienstleistungen auf den Umgang mit Kranken auswirken.

\section{Literatur}

1 Unschuld PU: Ware Gesundheit. Das Ende der klassischen Medizin, ed 3. München, C.H. Beck, 2014. 